\title{
Mitochondrial content and oxidative damage in full-term placentas from SGA, LGA and AGA infants pregnant women
}

\section{Type}

Research paper

\section{Keywords}

Placenta, Oxidative damage, Nitrosative damage, Mitochondrial content, Small for gestational age, Appropriate for gestational age, Large for gestational age

\begin{abstract}
Introduction

The aim was to determine the mitochondrial content, oxidative and nitrosative status in placentas from pregnant women who delivery newborns with alteration of intrauterine growth.

\section{Material and methods}

Placentas were selected because the newborns were classified as small for gestational age (SGA, lowest 10th percentile; $n=9)$, appropriate for gestational age (AGA; $n=9)$ and large for gestational age (LGA, tallest 90th percentile; $n=9$ ). In the placenta tissue oxidative and nitrosative status, and the mitochondrial content were determined.
\end{abstract}

\section{Results}

Lipid peroxidation (TBARS) levels were higher in LGA placentas compared with SGA placentas, but not compared with AGA placentas. Carbonyl levels were higher in LGA placentas compared with the AGA and SGA placentas. The 3-nitrotyrosine (3-NT)/actin ratio was higher in the SGA and LGA placentas than in AGA placentas. Moreover, AGA placentas did have higher cytochrome oxidase (COX4)/actin ratio compared with the SGA and LGA placentas. The AMP-activated protein kinase alpha (AMPK $\square \square /$ actin ratio was significantly lower in placentas from SGA compared with the placentas from AGA and LGA. With respect to the adenosine triphosphatase (ATPase) activity, this was significantly lower in placentas from LGA compared with the placentas from AGA and SGA.

\section{Conclusions}

The placentas of LGA newborns have higher oxidized lipid and protein levels, whereas SGA and LGA placentas have higher nitrosative damage levels than the AGA placentas; the present data also suggest that the mitochondrial content is lower in SGA and LGA placentas than in AGA placentas. 
Mitochondrial content and oxidative damage in full-term placentas from SGA, LGA and AGA infants pregnant women

Ramírez-Emiliano Joel*, Barbosa-Sabanero Gloria, Solís-Martínez Martha O., Mina-Bravo Mariana F., Martínez-Escamilla Edgar A. and Pérez-Vázquez Victoriano

Departamento de Ciencias Médicas, División de Ciencias de la Salud, Campus León, Universidad de Guanajuato. León, Gto., México. *E-mail: joelre@ugto.mx

Running head: Mitochondrial content and oxidative damage in human placenta 


\begin{abstract}
Introduction: The aim was to determine the mitochondrial content, oxidative and nitrosative status in placentas from pregnant women who delivery newborns with alteration of intrauterine growth.
\end{abstract}

Material and Methods: Placentas were selected because the newborns were classified as small for gestational age (SGA, lowest 10th percentile; $n=9$ ), appropriate for gestational age (AGA; $n$ =9) and large for gestational age (LGA, tallest 90th percentile; $n=9)$. In the placenta tissue oxidative and nitrosative status, and the mitochondrial content were determined.

Results: Lipid peroxidation (TBARS) levels were higher in LGA placentas compared with SGA placentas, but not compared with AGA placentas. Carbonyl levels were higher in LGA placentas compared with the AGA and SGA placentas. The 3-nitrotyrosine (3-NT)/actin ratio was higher in the SGA and LGA placentas than in AGA placentas. Moreover, AGA placentas did have higher cytochrome oxidase (COX4)/actin ratio compared with the SGA and LGA placentas. The AMP-activated protein kinase alpha $(\mathrm{AMPK} \alpha) /$ actin ratio was significantly lower in placentas from SGA compared with the placentas from AGA and LGA. With respect to the adenosine triphosphatase (ATPase) activity, this was significantly lower in placentas from LGA compared with the placentas from AGA and SGA.

Conclusions: The placentas of LGA newborns have higher oxidized lipid and protein levels, whereas SGA and LGA placentas have higher nitrosative damage levels than the AGA placentas; the present data also suggest that the mitochondrial content is lower in SGA and LGA placentas than in AGA placentas. 
Key words: Placenta; Oxidative damage; Nitrosative damage; Mitochondrial content; Small for gestational age; Appropriate for gestational age; Large for gestational age. 


\section{Introduction}

It has been suggested that the intrauterine environment is a key factor in intrauterine growth and subsequent metabolic development. Thus, an unhealthy environment may induce intrauterine growth disorders, resulting in newborns that are small for gestational age (SGA, birthweight $\leq 10$ th percentile) or large for gestational age (LGA, birthweight $\geq 90$ th percentile). This is a serious health problem because SGA and LGA infants have higher morbidity and mortality, and incur higher hospital charges than the newborns who are appropriate for gestational age (AGA) $[1,2]$. However, the etiology of the alteration of intrauterine growth is not completely understood, but it is thought to be related to maternal, fetal, and placental factors.

There may be a association between intrauterine growth disorders and bioenergetic imbalance, where the mitochondria play a key role in energy production. A report showed that the placenta of SGA newborns showed a significant decrease in enzymatic activities of both the mitochondrial respiratory chain and citrate synthase compared with the placentas of AGA newborns [2]. In the human placenta, mitochondrial DNA (mtDNA) abundance and oxidative damage (8-hydroxy-2' deoxyguanosine) are positively associated with intrauterine growth restriction (IUGR) [3], and the placentas of IUGR pigs had greater reactive oxygen species (ROS) production and oxidative injury with a concomitant reduction in mtDNA content [4]. In addition, vitamin $\mathrm{C}$ and $\mathrm{E}$ levels were significantly decreased in the serum of pregnant women with IUGR births compared to healthy pregnant women [5]. Importantly, in the guinea pig, supplementation with $\mathrm{N}$-acetylcysteine, a precursor of the antioxidant glutathione, normalized endothelial function in IUGR placenta and fetus, and consequently normalized fetal growth [6]. 
LGA human newborns are more likely to become obese adolescents compared with AGA newborns [7]; moreover, women with pregestational obesity have an increased risk of delivering LGA newborns at term compared with pregnant women with normal weight [8]. However, the mitochondrial content in the placentas of LGA newborns is unknown. Therefore, the present study aimed to determine the mitochondrial content and oxidative and nitrosative status in placental biopsies from healthy women who delivered SGA, AGA, and LGA infants at term.

\section{Material and Methods}

\section{Patients and sample collection}

A cross-sectional study was performed on two groups of patients. All the pregnant women were recruited from the General Regional Hospital in the city of León Gto., México, and the study was approved by the ethics committee of the General Regional Hospital (Reg. no. HGRL06022007). First, pregnant women were screened for preeclampsia, gestational or type 2 diabetes, antiphospholipid syndrome, connective tissue diseases, chronic infection, alcohol consumption or smoking habit during the current pregnancy, and all women participating in the study had a negative screen. We included mothers between 18 and 35 years of age and their term (37-40 weeks) newborns, born from vaginal delivery or non-elective cesarean section, without perinatal asphyxia or acute fetal suffering signs, and with an Apgar score of $\geq 7$ at the first minute. The mothers provided signed informed consent before inclusion into the study. Second, we included newborns that met the inclusion criteria according to their birthweight and gestational age (3740 weeks) using tables currently validated for the Mexican population [9]. Only 9 newborns of 
healthy mothers met the inclusion criteria for SGA. Therefore, the 3 groups were adjusted to 9 cases per group as follows: SGA (birthweight $\leq 10$ th percentile; $n=9$ ), AGA (11th-89th percentile; $n=9$ ), and LGA (birthweight $\geq 90$ th percentile; $n=9$ ), resulting in a total of 27 newborns. Although the sample size was relatively modest, the statistical power for the marker of mitochondrial content ( $\mathrm{COX} 4)$ was 0.97 , and that for the marker of oxidative damage (carbonyls) was 0.9 .

Placentas were obtained within 15 min of delivery and dissected fragments, including fetal and maternal sides, were stored at $-70{ }^{\circ} \mathrm{C}$ until further processing. Later, a placental lobule (cotyledon) was removed from the region next to umbilical cord, the basal plate and chorionic surface were removed from the cotyledon, and villous tissue was obtained from the middle cross section. Placental tissues were blunt dissected to remove visible connective tissue and calcium deposits as previously described [10]. Then, $100 \mathrm{mg}$ of the placental tissue were dissected and were homogenized in buffer (10 mM HEPES, $0.6 \%$ Nonidet p-40, $150 \mathrm{mM} \mathrm{NaCl}, 1 \mathrm{mM}$ ethylenediaminetetraacetic acid (EDTA) containing protease inhibitors (Complete, Boehringer Mannheim, Germany). Whole protein lysates were assayed for protein concentration using bicinchoninic acid (BCA) protein assay (Pierce Chemical Co., Rockford, IL, USA) with bovine serum albumin (BSA) as the reference standard.

\section{SDS-PAGE and western blotting}

Thirty $\mu \mathrm{g}$ of placenta lysate was separated on $10 \%$ polyacrylamide gel, and resolved proteins were transferred to nitrocellulose membrane. Then, the blots were probed with antibodies (Santa 
Cruz Biotechnology, Inc.) at the following dilutions: 3-nitrotyrosine 1:1500, Subunit IV of COX4 1:1500, AMPK $\alpha 1 / 2$ 1:800 and $\beta$-actin 1:3000. For more detailed description see Ramirez-Emiliano et al. 2017 [10].

\section{Determination of ATPase activity}

It is important to consider that the ATPase activity is an indirect indicator of mitochondrial content, and it does not rule out other activities that have been described in the placenta as nongastric $\mathrm{H}+/ \mathrm{K}+\mathrm{ATPase}[11], \mathrm{Na}^{+} / \mathrm{K}^{+}$-ATPase [12], and $\mathrm{Ca}^{2+}$ ATPase [13]. In the present study the ATPase activity was performed by determining the adenosine triphosphate (ATP) consumption using a High-performance liquid chromatography (HPLC) system as we previously described [10]. Moreover, the content of ATP in placentas was also determined and considered to calculate the ATPase activity, which is expressed as pmols of ATP consumed $/ \mathrm{mg} \operatorname{prot} \cdot \mathrm{min}$.

\section{Measurement of lipid peroxidation and oxidized protein}

The most common way to measure the levels of lipid peroxidation is to estimate malondialdehyde (MDA) content by the thiobarbituric acid-reactive substances (TBARS) assay, whereas the oxidized proteins are quantified by measuring the carbonyls content. Therefore, the total homogenate of placenta tissue were used to quantify the MDA levels by the TBARS assay, and the carbonyls content as we previously described $[10,14,15]$. 


\section{Statistical analysis}

The statistical analyses were performed with Statistics for Windows 8 (StatSoft, Inc.). Data were analyzed with ANOVA followed by post hoc tests to find the differences between groups. The significance level set at $\mathrm{p}<0.05$.

\section{Results}

\section{Anthropometric characteristics of participants}

The anthropometric characteristics of the newborns are shown in Table 1. The newborns were classified based on their birthweight and gestational age using the tables currently validated for the Mexican population [9] (10th, 11-89th and 90th percentiles). LGA newborns had a higher birthweight $(p<0.01)$ and were longer $(p<0.05)$ than the SGA and AGA newborns, and the AGA group had a higher birthweight $(p<0.05)$ and were longer $(p<0.05)$ than the SGA group.

\section{Mitochondrial content in the placenta}

To indirectly analyze the mitochondrial content, the expression levels of COX4 and AMPK, and the ATPase activity were determined. The COX4, AMPK, and actin protein levels were determined by western blotting; thereafter, variations in the expression levels were corrected by determining the COX4, AMPK, and 3-NT/actin ratios. As shown in Figures 1a and b, COX4/actin ratios were significantly lower $(p<0.01)$ in placentas from the SGA and LGA groups $(0.25 \pm 0.1$ and $0.62 \pm 0.08$, respectively) than the placentas from the AGA group (1.08 \pm 0.1). Moreover, the COX4/actin ratio was significantly lower $(p<0.05)$ in placentas from the 
SGA group than in placentas from the LGA group. No significant differences were observed in AMPK/actin ratios between the placentas from the AGA and LGA groups $(0.2 \pm 0.03$ and $0.22 \pm$ 0.04, respectively; Fig. 1a and 1c). However, the AMPK/actin ratio was significantly lower in placentas from the SGA group $(0.12 \pm 0.01, p<0.05)$ compared with the placentas from the AGA and LGA groups. ATPase activity was similar in the placentas from the AGA and SGA groups (11.86 \pm 1.6 and $10.7 \pm 0.9 \mathrm{pmols}$ of ATP consumed/mg prot.min, respectively), but was significantly lower in placentas from the LGA group $(8 \pm 0.6 \mathrm{pmols}$ of ATP consumed $/ \mathrm{mg}$ prot.min, $p<0.05$ ) compared with the placentas from the AGA and SGA groups (Fig. 2).

\section{Oxidative and nitrosative damage in placentas}

The levels of TBARS were lower in SGA group placentas than in AGA group placentas ( $4.6 \pm$ 0.4 vs. $6.2 \pm 0.5 \mathrm{nmoles} / \mathrm{mg}$ protein, respectively), but this difference was not statistically significant. In contrast, TBARS levels were higher in LGA group placentas than in SGA group placentas (7.3 \pm 0.9 nmoles $/ \mathrm{mg}$ protein, $p<0.01)$ (Fig. 3a). Moreover, the carbonyls levels were higher in LGA group placentas $(1048.6 \pm 83 \mathrm{ng} / \mathrm{mg}$ protein, $p<0.01)$ than in the placentas from the AGA and SGA groups $(583.6 \pm 106.8$ and $673.6 \pm 83$ nmoles/mg protein, respectively; Fig. 3b). No significant differences were observed between the placentas from the AGA and SGA groups.

With respect to nitrosative damage, the levels of nitrated proteins were determined by measuring the 3-nitrotyrosine (3-NT) content by western blotting, as shown in the Figure 4a; thereafter, the 3-NT/actin ratios were determined. The 3-NT/actin ratio was higher in placentas from the SGA 
group $(2.7 \pm 0.6, p<0.05)$ than in placentas from the AGA group $(1.2 \pm 0.09)$, but these ratios were similar in the placentas from the AGA and LGA groups $(1.2 \pm 0.09$ vs. $1.7 \pm 0.14, p>0.05)$ (Fig. 4b). The 3-NT/actin ratio was higher in placentas in the SGA group than in placentas from the LGA group, but this difference was not statistically significant (Fig. 4b).

\section{Discussion}

In the Mexican population, intrauterine growth disorders are classified as SGA and LGA in comparison with AGA, based on birthweight and gestational age [9]. The present study aimed to compare the mitochondrial content and oxidative and nitrosative damage levels in placentas of healthy women who delivered at term, and whose newborns were from vaginal delivery or nonelective cesarean section. We observed low expression levels of COX4 and AMPK and decreased ATPase activity, suggesting that the placentas of SGA and LGA newborns have less mitochondrial content compared with the placentas of AGA newborns; however, it is important to consider that the ATPase activity that we determined represents the activity of all the ATPases in the placenta. The low expression levels of COX4 observed in the present study suggest decreased complex IV activity, which is important because a low complex IV activity is closely associated with a decrease in the corresponding mitochondrial oxidative phosphorylation capacity [16]. In addition, AMPK is important for inducing mitochondrial biogenesis [17]; therefore, the low expression levels of AMPK observed in the present study also suggest a reduction in mitochondrial biogenesis in the placentas of SGA newborns. The low ATPase activity observed in the present study also supports the hypothesis that the placentas of SGA and LGA newborns have reduced mitochondrial content compared with the placentas of AGA 
newborns. Other studies support these findings; Guitar-Mampel et al. showed a reduction in the enzymatic activities of mitochondrial respiratory chain complexes I and citrate synthase in the placentas of SGA newborns [2]. In another study, the expression of genes involved in three complexes of the mitochondrial respiratory chain was decreased in IUGR placentas [18]. In addition to the above, low mtDNA content and mtDNA-encoded gene expression was also observed in placentas of IUGR pig fetus, suggesting a reduction of the mitochondrial content. Further, in another study, the activation of MAPK pathways was observed because the ERK1/2 phosphorylation was increased whereas the phosphorylation of p38 and JNK was decreased [4], possibly because the activation of MAPK pathways is a response to an increase in the low mitochondrial biogenesis observed in these placentas. Interestingly, low messenger RNA (mRNA) levels of complex II, III, and IV were observed in IUGR cytotrophoblast cells but with no differences at the protein level, which suggests a posttranscriptional compensatory regulation. In contrast, mtDNA was increased in the placentas of IUGR infants [19].

Children who were LGA at birth and were exposed to an intrauterine environment of maternal diabetes or obesity are at an increased risk of developing metabolic syndrome [20], and maternal obesity is also associated with an increased risk of delivering LGA newborns, resulting in an increased obesity risk in early childhood [21]. In addition, Rosado-Yépez et al. also showed that women with pregestational obesity have an increased risk of delivering at term newborns that are LGA compared with normal-weight women, and the placentas of LGA infants had a lower placental maturity index $\left(\mathrm{PMI}=\right.$ number of vasculo-syncytial membranes $[\mathrm{VSM}]$ in $1 \mathrm{~mm}^{2} / \mathrm{VSM}$ thickness) [8]. Moreover, Lekva et al. reported that serum adiponectin was lower in mothers who gave birth to LGA newborns, whereas adiponectin receptor 2 and system A amino acid transporter mRNA expression were lower in the placentas of LGA infants [22]. Together, these 
findings show that the obesogenic intrauterine environment increases the risk of LGA newborns; however, there is no evidence regarding the mitochondrial content in placentas and LGA newborns. Thus, to our knowledge, our study is the first to assess and compare the mitochondrial content in placentas of SGA, AGA and LGA newborns.

It is widely known that oxidative and nitrosative injuries are involved in the dysfunction of organelles and tissues. As described above, the TBARS levels were significantly higher in the in placentas of LGA newborns than in those of SGA newborns, with these levels being intermediate in AGA-newborn placentas. Importantly, the carbonyl levels were congruent with the TBARS levels, because the LGA group placentas had higher carbonyl levels than the placentas from the AGA and SGA groups. In all, these findings show that the placentas of LGA newborns experience more oxidative damage than the placentas of SGA newborns, possibly because the former placentas have a higher energetic demand than the latter placentas. It is reasonable to assume that a large fetus requires more energy than a small fetus; therefore, the placentas of LGA fetuses must increase their metabolism to meet the energy requirements of the fetus and placenta. It is well known that tissues with higher energy demand are more oxidized; for example, the metabolism in the liver is higher than that in the kidney, and TBARS and carbonyl levels are higher in the liver than in the kidney [23]. In contrast to that, Lou et al. found that in pig placentas, ROS production and lipid peroxidation were increased in the placentas of IUGR pigs compared with those of AGA pigs; however, they did not analyze the placentas of LGA pigs [4].

With respect to nitrosative damage, the 3-NT content was surprisingly higher in placentas from SGA newborns than in the placentas from AGA and LGA newborns, and no significant 
differences were found between placentas from AGA and LGA newborns. This nitrosative damage profile differs with respect to the profile of oxidative damage. These findings are in concordance with previously reported data, which showed that oxidative damage might decrease, whereas nitrosative damage may increase in the placentas of women with gestational diabetes mellitus [10].

It is important to note that in the present study, the causes of the intrauterine growth disorders were not identified. Other studies have been conducted to determine the etiology of intrauterine growth disorders, and it is not clear how or when these disorders occur. However, a retrospective population-based cohort study described that women with Müllerian anomalies have an increased risk (odds ratio, 1.9) of delivering newborns with IUGR [24]. In a study, overweight and obese women with gestational diabetes mellitus had maternal vascular malperfusion lesions in the placenta, and these lesions were associated with a 2-fold increased risk for the delivery of an SGA newborn [25]. With respect to LGA newborns, a cross-sectional study including Mexican women showed that the weight and length of newborns at birth were greater in women with pregestational obesity than in women with normal weight [8]. In Iranian pregnant women, a significant correlation was found between the increase in fasting plasma glucose, triglyceride (TG), and TG/HDL-C ratio and the risk of gestational diabetes mellitus and LGA infant occurrence in the first trimester [26]. This was previously suggested by Gutaj et al., who described that the decreased HDL and increased triglycerides during pregnancy might contribute to the development of LGA infants in women with type 1 diabetes [27]. Moreover, the LGA newborns of women with a high BMI demonstrated higher levels of leptin compared to LGA 
newborns of mothers with a normal BMI, whereas leptin levels in AGA neonates were similar regardless of the nutritional status of their mothers [28].

There are some strengths and limitations of the present study. With regard to the study strengths, although the newborns in the study presented with intrauterine growth disorders, their mothers were healthy, which reduced the population variability. Further, to our knowledge, this is the first study to assess and compare the mitochondrial content in placentas of SGA, AGA, and LGA newborns. However, our study was limited by its small sample size, we did not separately analyze the fetal and maternal sides of the placenta, and the causes of the intrauterine growth disorders were not identified.

The present results have some implications for clinicians. First, because intrauterine growth disorders are accompanied by oxidative stress, antioxidant supplementation is important in the diet of pregnant women whose fetus have such disorders. Moreover, it is important to determine the total antioxidant status, total oxidant status, and oxidative stress index values in the serum of pregnant women in order to stablish the severity of oxidative stress.

Finally, the present results need to be further validated by: (1) additional studies including a greater number of patients, (2) obtainning results regarding the two sides of the placenta, (3) determining the correlation between intrauterine growth disorders and their etiology, and (4) determining the effectiveness of treatment with antioxidant supplements for intrauterine growth disorders. Moreover, ascertaining when there may be a shift in the balance between nitrosative and oxidative stress is desirable. Further, in the newborn, long-term follow-up is crucial to 
determine the clinical consequences of the reduction of the mitochondrial content and of nitrosative and oxidative damage in the placenta.

\section{Conclusions}

Our findings strongly suggest that the oxidative damage in placentas is proportional to birthweight because the placentas of LGA newborn had higher TBARS and carbonyl levels than the placentas of SGA newborns. The placentas of SGA and LGA newborns were more susceptible to nitrosative damage than AGA placentas. Moreover, the present results suggest that both mitochondrial biogenesis and content are decreased in the placentas of SGA and LGA newborns.

\section{Abbreviations}

IUGR, Intrauterine growth restriction; SGA, small for gestational age; AGA, appropriate for gestational age; LGA, large for gestational age; ROS, reactive oxygen species; mtDNA, mitochondrial DNA; ATP, Adenosine triphosphate; AMP, Adenosine 5'-monophosphate; AMPK, AMP-activated protein kinase; TBARS, Thiobarbituric acid-reactive substances; 3-NT, 3-nitrotyrosine; COX4, Subunit IV of cytochrome oxidase; HDL, high-density lipoprotein; LDL, low-density lipoprotein.

\section{Declarations}




\section{Ethics approval and consent to participate}

The study was performed in accordance with the Declaration of Helsinki of the World Medical Association, who sets the standards for research involving human subjects; moreover, this was approved by the ethics committee of the ethics committees of the hospital (Reg. no.

HGRL06022007). All patients signed an informed consent form for this investigation.

\section{Availability of data and materials}

The datasets used and/or analyzed during the current study are available from the corresponding author on reasonable request.

\section{Consent for publication}

Not applicable.

\section{Conflict of interest}

The authors declare that they have no conflict of interest.

\section{Funding}


Funding for this study was provided by the CONACYT (I010/532/2014) to Ramírez-Emiliano Joel.

\section{Authors' contributions}

JRE and GBS, designed the study, wrote and reviewed the paper critically, and approved the manuscript. MOSM, MFMB and EAME performed the study and analyzed the data. VPV analyzed the data, reviewed the paper critically, and approved the manuscript.

\section{Acknowledgments}

The authors wish to thank the pregnancies women, who made this study possible, the doctors and nurses of the Hospital General Regional de León for their trust in allowing their facilities to help perform this study.

\section{References}

1. Ewing AC, Ellington SR, Shapiro-Mendoza CK, Barfield WD, Kourtis AP. Full-term smallfor-gestational-age newborns in the U.S.: characteristics, trends, and morbidity. Matern Child Health J 2017; 21(4): 786-96.

2. Guitart-Mampel M, Juarez-Flores DL, Youssef L, et al. Mitochondrial implications in human pregnancies with intrauterine growth restriction and associated cardiac remodelling. $\mathbf{J}$ Cell Mol Med 2019; 23(6): 3962-73. 
3. Jones R, Peña J, Mystal E, et al. Mitochondrial and glycolysis-regulatory gene expression profiles are associated with intrauterine growth restriction. J Matern Fetal Neonatal Med 2020; 33(8): 1336-45.

4. Luo Z, Luo W, Li S, et al. Reactive oxygen species mediated placental oxidative stress, mitochondrial content, and cell cycle progression through mitogen-activated protein kinases in intrauterine growth restricted pigs. Reprod Biol 2018; 18(4): 422-31.

5. Gadhok AK, Sharma TK, Sinha M, et al. Natural antioxidant vitamins status in pregnancies complicated with intrauterine growth restriction. Clin Lab 2017; 63(5): 941-5.

6. Herrera EA, Cifuentes-Zúñiga F, Figueroa E, et al. N-Acetylcysteine, a glutathione precursor, reverts vascular dysfunction and endothelial epigenetic programming in intrauterine growth restricted guinea pigs. J Physiol 2017; 595(4): 1077-92.

7. Vohr BR, Lipsitt LP, Oh W. Somatic growth of children of diabetic mothers with reference to birth size. J Pediatr 1980; 97(2): 196-9.

8. Rosado-Yépez PI, Chávez-Corral DV, Reza-López SA, et al. Relation between pregestational obesity and characteristics of the placenta. J Matern Fetal Neonatal Med 2019: 1-6.

9. Jurado-Garcia E. [Intrauterine growth. Correlation of body weight-height at birth as a function of gestational age]. Gac Med Mex 1971; 102(2): 227-55.

10. Ramirez-Emiliano J, Fajardo-Araujo ME, Zuniga-Trujillo I, Perez-Vazquez V, SandovalSalazar C, Ornelas-Vazquez JK. Mitochondrial content, oxidative, and nitrosative stress in human full-term placentas with gestational diabetes mellitus. Reprod Biol Endocrin 2017; 15. 
11. Johansson M, Jansson T, Pestov NB, Powell TL. Non-gastric H+/K+ ATPase is present in the microvillous membrane of the human placental syncytiotrophoblast. Placenta 2004; 25(6): 505-11.

12. Floyd RV, Wray S, Martin-Vasallo P, Mobasheri A. Differential cellular expression of FXYD1 (phospholemman) and FXYD2 (gamma subunit of Na, K-ATPase) in normal human tissues: A study using high density human tissue microarrays. Ann Anat 2010; 192(1): 7-16.

13. Yang H, Kim TH, An BS, et al. Differential expression of calcium transport channels in placenta primary cells and tissues derived from preeclamptic placenta. Mol Cell Endocrinol $2013 ; 367(1-2): 21-30$.

14. Karabekir HS, Guzey FK, Balci C, et al. Biochemical changes representing oxidative stress on brain tissue due to intraabdominal hypertension in a rat model. Arch Med Sci 2007; 3(3): 215-22.

15. Vujotić L, Matić S, Borković-Mitić S, et al. Association between oxidative stress biomarkers and concentrations of some metal ions in the blood of patients with brain tumors and hydrocephalus. Arch Med Sci 2019.

16. Larsen S, Nielsen J, Hansen CN, et al. Biomarkers of mitochondrial content in skeletal muscle of healthy young human subjects. J Physiol 2012; 590(14): 3349-60.

17. Kluge MA, Fetterman JL, Vita JA. Mitochondria and endothelial function. Circ Res 2013; 112(8): 1171-88.

18. Madeleneau D, Buffat C, Mondon F, et al. Transcriptomic analysis of human placenta in intrauterine growth restriction. Pediatr Res 2015; 77(6): 799-807. 
19. Mandò C, De Palma C, Stampalija T, et al. Placental mitochondrial content and function in intrauterine growth restriction and preeclampsia. Am J Physiol Endocrinol Metab 2014; 306(4): E404-E13.

20. Boney CM, Verma A, Tucker R, Vohr BR. Metabolic syndrome in childhood: association with birth weight, maternal obesity, and gestational diabetes mellitus. Pediatrics 2005; 115(3): e290-6.

21. Pringle KG, Lee YQ, Weatherall L, et al. Influence of maternal adiposity, preterm birth and birth weight centiles on early childhood obesity in an Indigenous Australian pregnancythrough-to-early-childhood cohort study. J Dev Orig Health Dis 2019; 10(1): 39-47.

22. Lekva T, Paasche Roland MC, Michelsen AE, et al. Large reduction in adiponectin during pregnancy is associated with large-for-gestational-age newborns. J Clin Endocrinol Metab 2017; 102(7): 2552-9.

23. Martinez-Morua A, Soto-Urquieta MG, Franco-Robles E, et al. Curcumin decreases oxidative stress in mitochondria isolated from liver and kidneys of high-fat diet-induced obese mice. J Asian Nat Prod Res 2013; 15(8): 905-15.

24. Cahen-Peretz A, Sheiner E, Friger M, Walfisch A. The association between Mullerian anomalies and perinatal outcome. J Matern-Fetal Neo M 2019; 32(1): 51-7.

25. Scifres CM, Parks WT, Feghali M, Caritis SN, Catov JM. Placental maternal vascular malperfusion and adverse pregnancy outcomes in gestational diabetes mellitus. Placenta 2017; 49: 10-5.

26. Pazhohan A, Rezaee Moradali M, Pazhohan N. Association of first-trimester maternal lipid profiles and triglyceride-glucose index with the risk of gestational diabetes mellitus and large for gestational age newborn. J Matern Fetal Neonatal Med 2019; 32(7): 1167-75. 
27. Gutaj P, Wender-Ozegowska E, Brazert J. Maternal lipids associated with large-forgestational-age birth weight in women with type 1 diabetes: results from a prospective single-center study. Arch Med Sci 2017; 13(4): 753-9.

28. Biesiada LA, Glowacka E, Krekora M, Sobantka S, Krokocka A, Krasomski G. The impact of excessive maternal weight on the nutritional status of the fetus - the role of leptin. Arch Med Sci 2016; 12(2): 394-401.

\section{Legends of Figures}

Figure 1. Expression levels of the COX4 and AMPK protein in placentas. A) Representative western blotting of the COX4, AMPK and actin. Densitometry analysis of the COX4/actin ratio (A) and AMPK/actin ratio (C). Data are given as the means \pm standard error (n=9). AGA, appropriate for gestational age; SGA, small for gestational age; LGA, large for gestational age. ${ }^{*} p<0.01$ vs. SAG and LGA; ${ }^{*} p<0.05$ vs. SAG; ${ }^{*} p<0.05$ vs. AGA and LGA.

Figure 2. ATPase activity in placentas. Data are given as the means \pm standard error $(n=9)$. AGA, appropriate for gestational age; SGA, small for gestational age; LGA, large for gestational age. ${ }^{*} p<0.05$ vs. AGA and SGA. 
Figure 3. (A) TBARS and (B) carbonyl levels in placentas. Data are given as the means \pm standard error $(n=9)$. AGA, appropriate for gestational age; SGA, small for gestational age; LGA, large for gestational age. ${ }^{*} p<0.01$ vs. SGA; ${ }^{*} p<0.001$ vs. NPW.

Figure 4. Nitrosative damage in placentas. A) Representative western blotting of the 3nitrotyrosine (3-NT) and actin. B) Densitometry analysis of the 3-NT/actin ratio. Data are given as the means \pm standard deviation $(n=9)$. AGA, appropriate for gestational age; SGA, small for gestational age; LGA, large for gestational age. ${ }^{*} p<0.05$ vs. AGA. 
Table 1. Newborns characteristics.

\begin{tabular}{lccc}
\hline & SGA & AGA & LGA \\
\hline Birth weight $(\mathbf{g})$ & $2413 \pm 162$ & $2887 \pm 260$ & $3584.4 \pm 443.6$ \\
Birth length $(\mathbf{c m})$ & $47.4 \pm 1.1$ & $49.3 \pm 1.2$ & $51.9 \pm 1.4$ \\
Gender (male/female) & $0 / 9$ & $4 / 5$ & $5 / 4$ \\
\hline
\end{tabular}

SGA, Small for gestational age; AGA, Appropriate for gestational age;

LGA, Large for gestational age. 
A

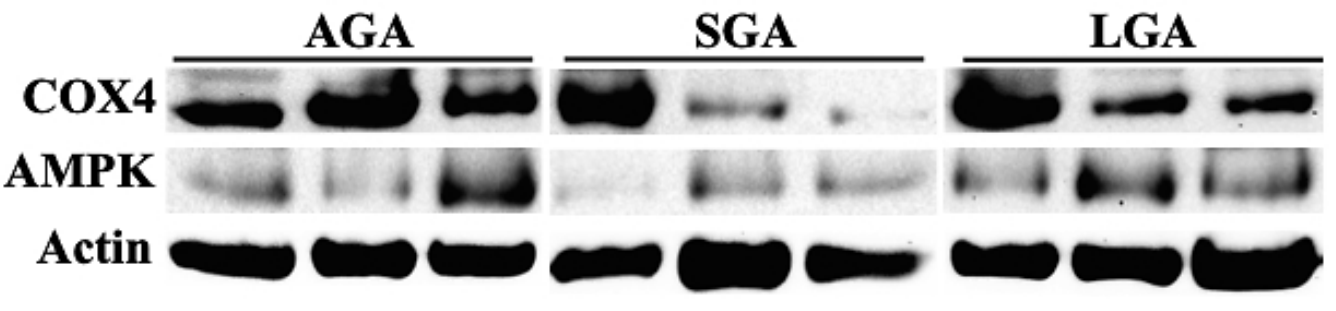

B

C
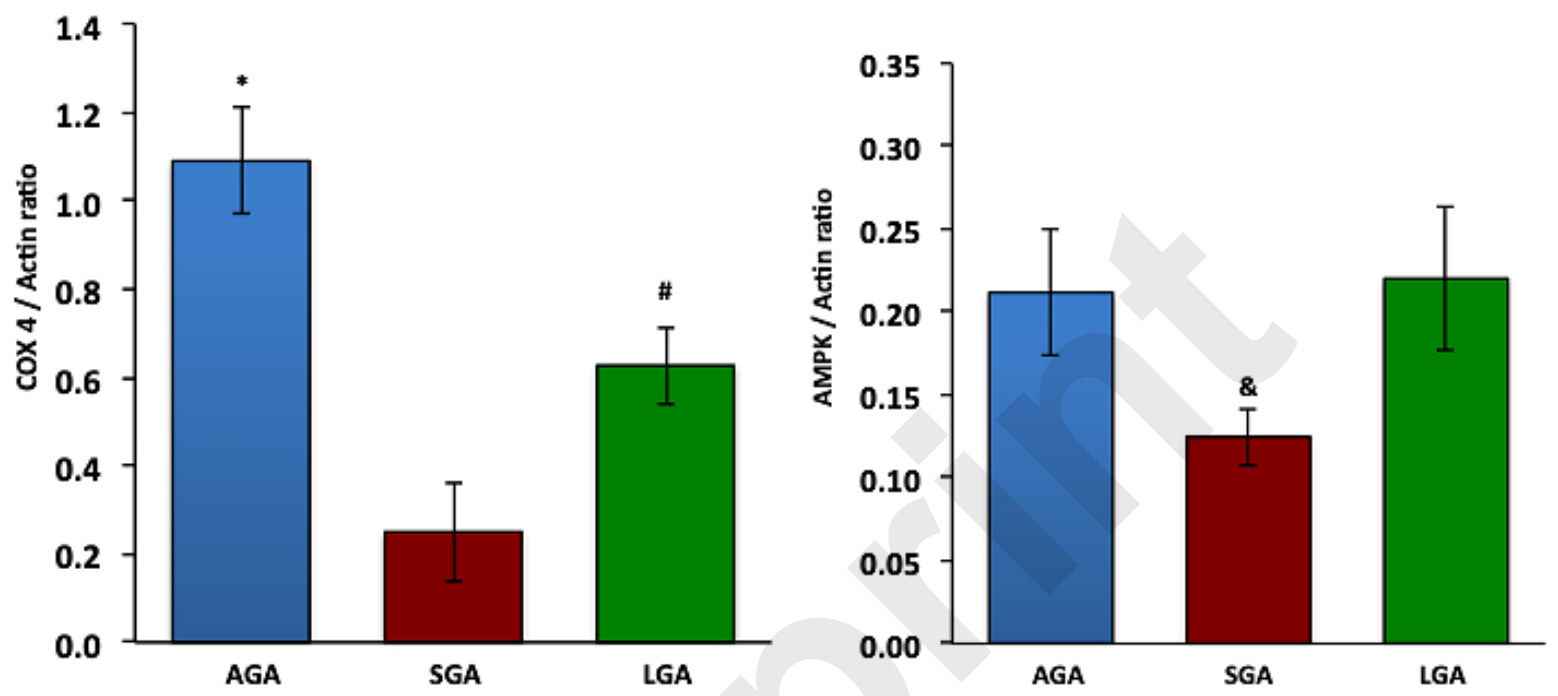


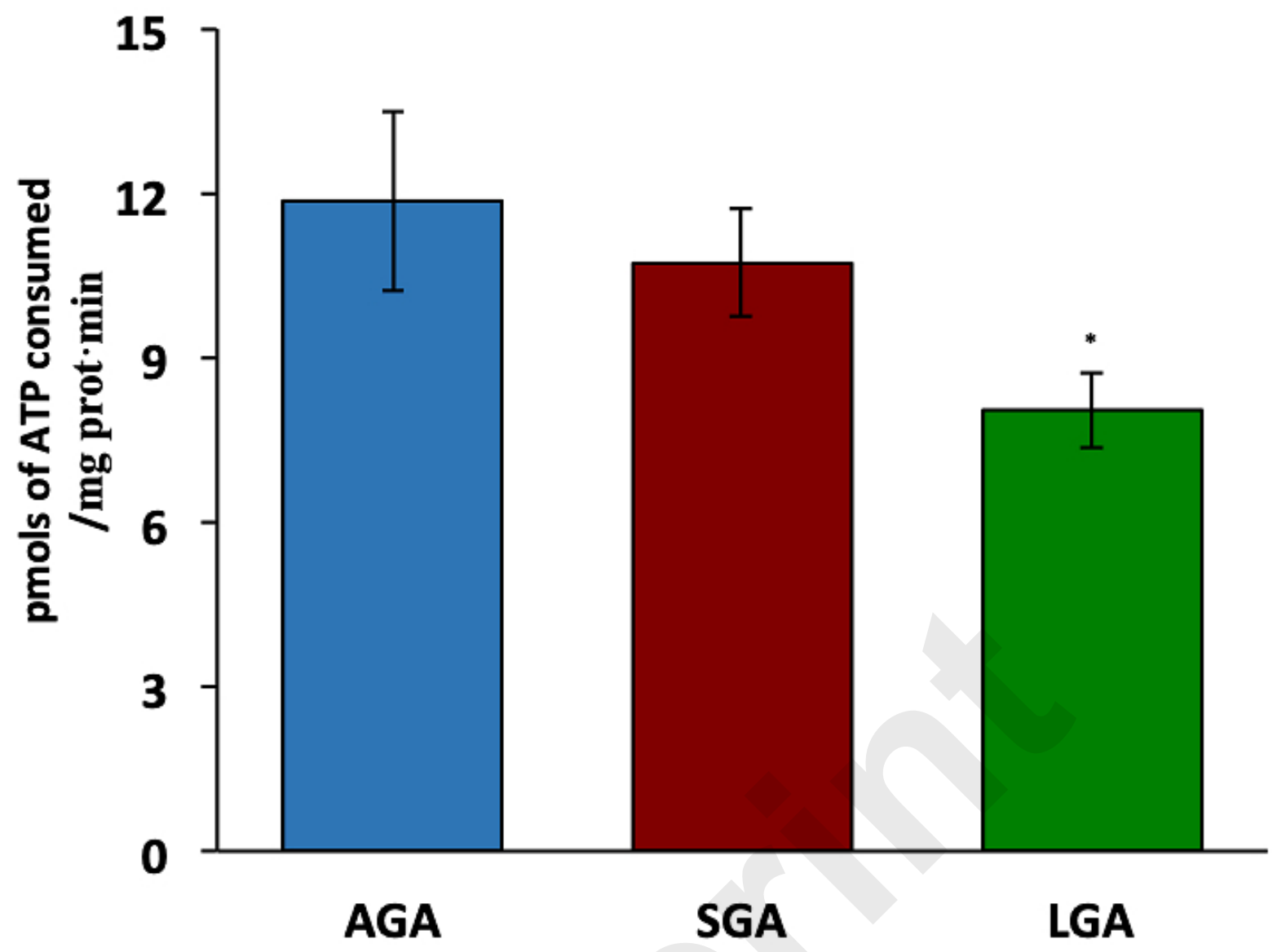


A

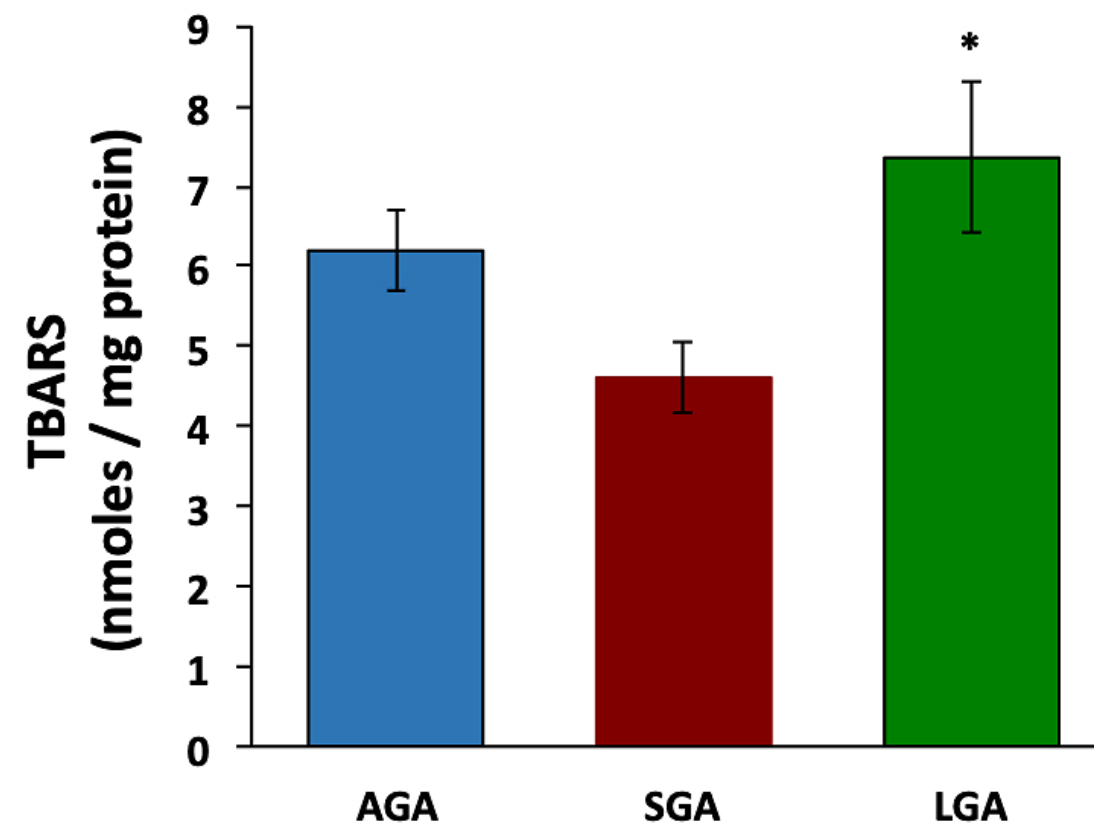

B

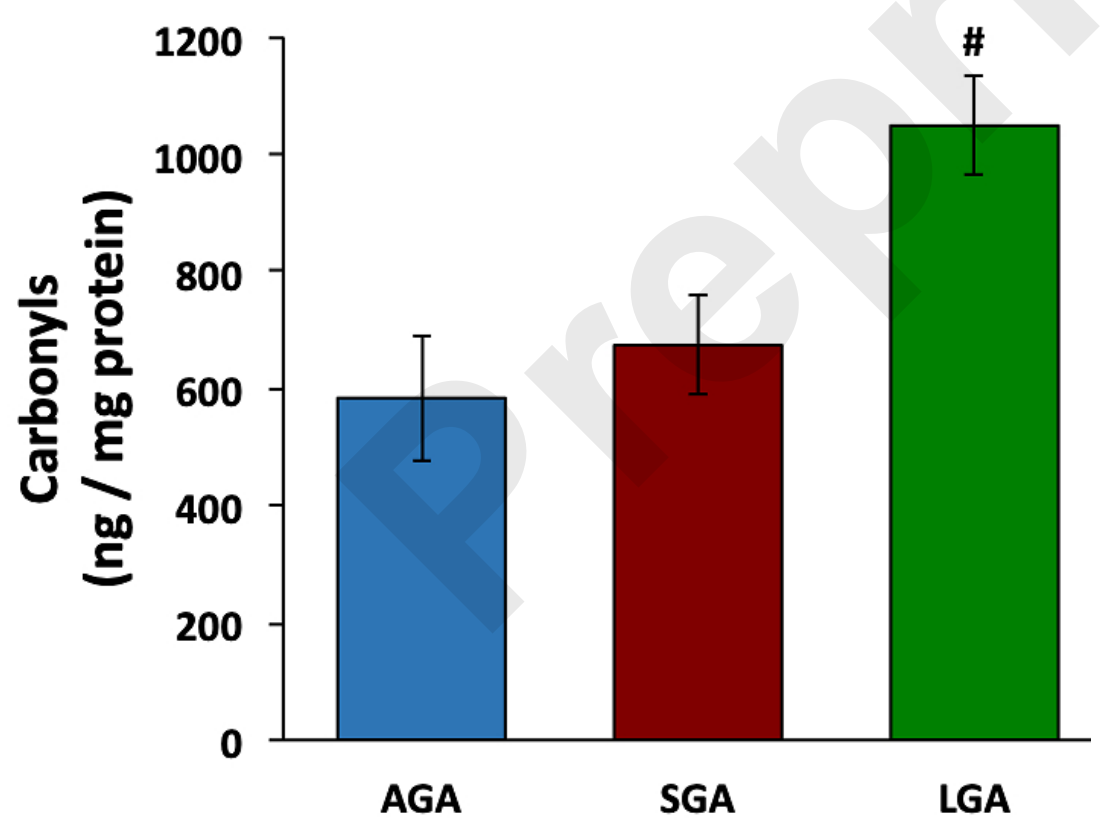


A

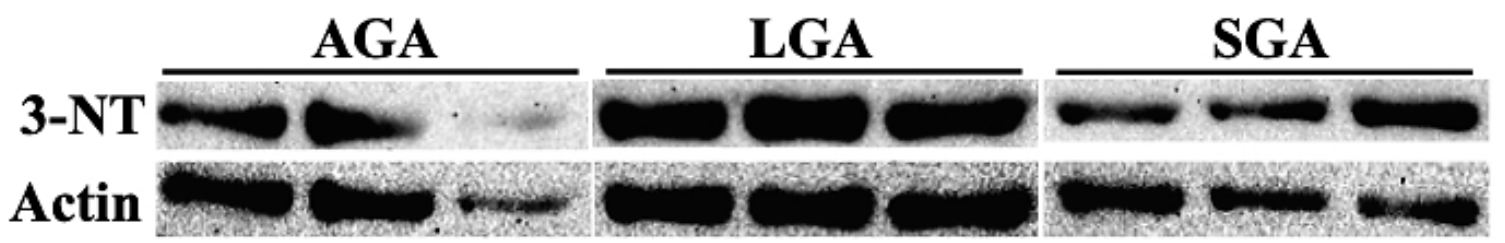

B

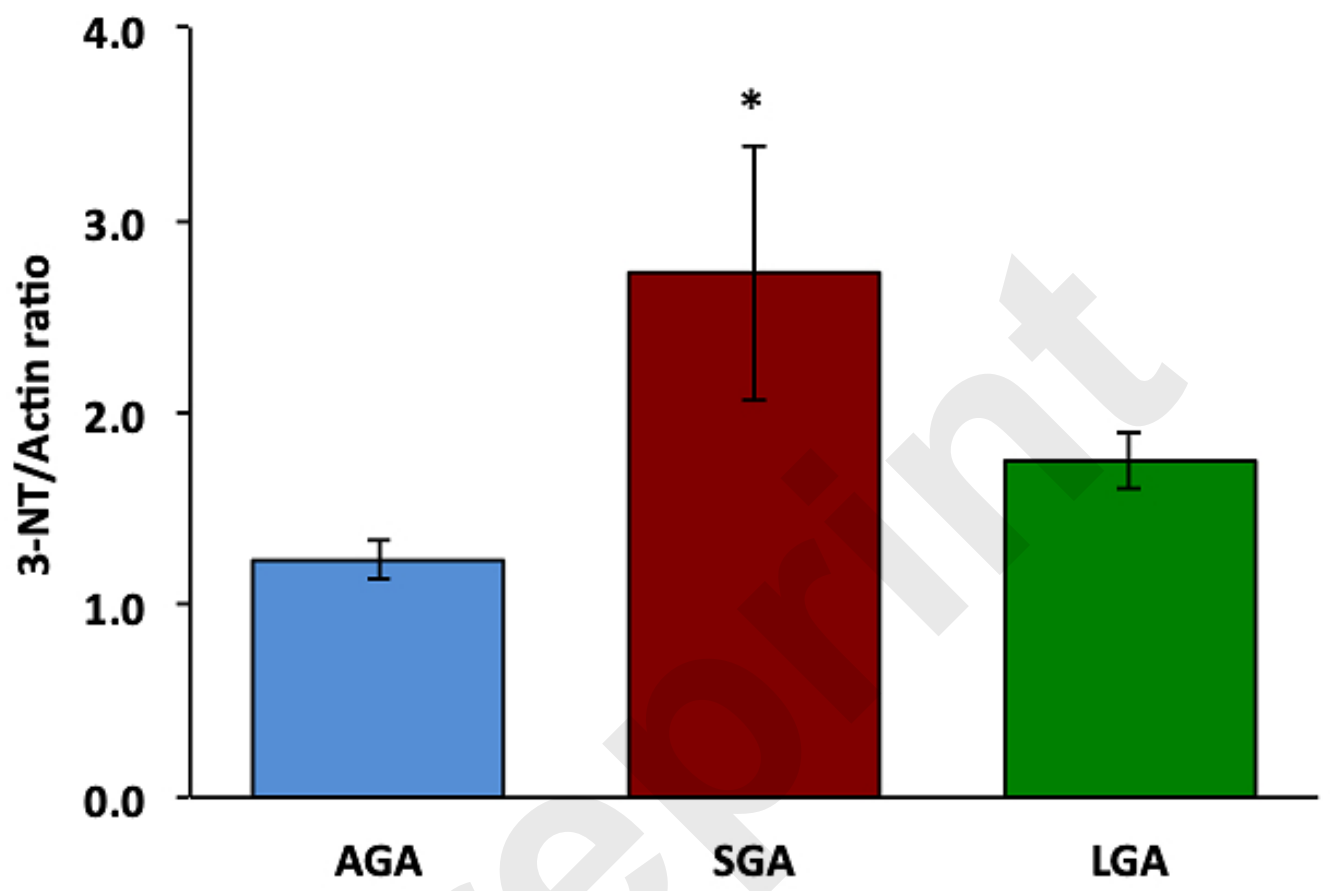

